\title{
Self Sustained Traversable Wormholes and Topology Change Induced by Gravity's Rainbow
}

\author{
Remo Garattini
}

\begin{abstract}
We consider the effects of Gravity's Rainbow on the self-sustained equation which is responsible to find new traversable wormholes configurations which are sustained by their own gravitational quantum fluctuations. The same selfsustained equation is also used to discover if topology change is possible. In this contribution, we will show that in both uses, the self-sustained equation will produce a Wheeler wormhole, namely a wormhole of Planckian size. This means that, from the point of view of traversability, the wormhole will be traversable in principle, but not in practice. From the topology change point of view, the background metric will be fixed to be Minkowskian in the equation governing the quantum fluctuations, which behaves essentially as a backreaction equation, and the quantum fluctuations are let to evolve. Analyzing this procedure, we will show that the self-sustained equation, endowed with a Gravity's Rainbow distortion, will be responsible of a topology change with the appearance of a Planckian wormhole.
\end{abstract}

\section{Introduction}

A wormhole is often termed Einstein-Rosen bridge because a "bridge" connecting two "sheets" was the result obtained by A. Einstein and N. Rosen in attempting to build a geometrical model of a physical elementary "particle" that was everywhere finite and singularity free[1]. It was J.A. Wheeler who introduced the term wormhole[2], although his wormholes were at the quantum scale. We have to wait for M. S. Morris and K. S. Thorne[3] to see the subject of wormholes seriously considered by the scientific community. In practice a traversable wormhole is a solution of the Einstein's Field equations, represented by two asymptotically flat re-

Remo Garattini

Università degli Studi di Bergamo, Dipartimento di Ingegneria,

Viale Marconi,5 24044 Dalmine (Bergamo) ITALY

I.N.F.N. - sezione di Milano, Milan, Italy, e-mail: remo.garattini@unibg.it 
gions joined by a bridge or, in other word, it is a short-cut in space and time. To exist, traversable wormholes must violate the null energy conditions, which means that the matter threading the wormhole's throat has to be "exotic". Classical matter satisfies the usual energy conditions. Therefore, it is likely that wormholes must belong to the realm of semiclassical or perhaps a possible quantum theory of the gravitational field. Since a complete theory of quantum gravity has yet to come, it is important to approach this problem semiclassically. On this ground, the Casimir energy on a fixed background. has the correct properties to substitute the exotic matter: indeed, it is known that, for different physical systems, Casimir energy is negative. Usually one considers some matter or gauge fields which contribute to the Casimir energy necessary to the traversability of the wormholes, nevertheless nothing forbids to use the Casimir energy of the graviton on a background of a traversable wormhole. In this way, one can think that the quantum fluctuations of the gravitational field of a traversable wormhole are the same ones which are responsible to sustain traversability. Nevertheless, Casimir energy is a form of Zero Point Energy (ZPE) which, usually manifests Ultra Violet (UV) divergences. To keep under control the UV divergences, usually one invokes a standard regularization/renormalization process. However, an alternative procedure can be taken under consideration by distorting spacetime since the beginning. This distortion is better known as Gravity's Rainbow. Since Gravity's Rainbow switches on at the Planck scale it is likely that ZPE can be used as a tool to produce a topology change. Note that in Ref.[4], the ZPE was used as an indicator for a topology change without a Gravity's Rainbow scheme. In this contribution we will explicitly show how Gravity's Rainbow comes into play to produce a topology change as a ZPE consequence.

\section{Self-sustained Traversable Wormholes}

In this Section we shall consider the formalism outlined in detail in Refs. [6, 7], where the graviton one loop contribution to a classical energy in a wormhole background is used. The spacetime metric representing a spherically symmetric and static wormhole is given by

$$
d s^{2}=-e^{2 \Phi(r)} d t^{2}+\frac{d r^{2}}{1-b(r) / r}+r^{2}\left(d \theta^{2}+\sin ^{2} \theta d \phi^{2}\right),
$$

where $\Phi(r)$ and $b(r)$ are arbitrary functions of the radial coordinate, $r$, denoted as the redshift function, and the shape function, respectively [3]. The radial coordinate has a range that increases from a minimum value at $r_{0}$, corresponding to the wormhole throat, to infinity. A fundamental property of a wormhole is that a flaring out condition of the throat, given by $\left(b-b^{\prime} r\right) / b^{2}>0$, is imposed [3, 5], and at the throat $b\left(r_{0}\right)=r=r_{0}$, the condition $b^{\prime}\left(r_{0}\right)<1$ is imposed to have wormhole solutions. Another condition that needs to be satisfied is $1-b(r) / r>0$. For the wormhole to be traversable, one must demand that there are no horizons present, which are iden- 
tified as the surfaces with $e^{2 \Phi} \rightarrow 0$, so that $\Phi(r)$ must be finite everywhere. The classical energy is given by

$$
H_{\Sigma}^{(0)}=\int_{\Sigma} d^{3} x \mathscr{H}^{(0)}=-\frac{1}{16 \pi G} \int_{\Sigma} d^{3} x \sqrt{g} R
$$

where the background field super-hamiltonian, $\mathscr{H}^{(0)}$, is integrated on a constant time hypersurface. $R$ is the curvature scalar, and using metric (11), is given by

$$
R=-2\left(1-\frac{b}{r}\right)\left[\Phi^{\prime \prime}+\left(\Phi^{\prime}\right)^{2}-\frac{b^{\prime}}{r(r-b)}-\frac{b^{\prime} r+3 b-4 r}{2 r(r-b)} \Phi^{\prime}\right]
$$

We shall henceforth consider a constant redshift function, $\Phi^{\prime}(r)=0$, which provides interestingly enough results, so that the curvature scalar reduces to $R=2 b^{\prime} / r^{2}$. Thus, the classical energy reduces to

$$
H_{\Sigma}^{(0)}=-\frac{1}{2 G} \int_{r_{0}}^{\infty} \frac{d r r^{2}}{\sqrt{1-b(r) / r}} \frac{b^{\prime}(r)}{r^{2}} .
$$

A traversable wormhole is said to be "self sustained" if

$$
H_{\Sigma}^{(0)}=-E^{T T},
$$

where $E^{T T}$ is the total regularized graviton one loop energy. Basically this is given by

$$
E^{T T}=-\frac{1}{2} \sum_{\tau}\left[\sqrt{E_{1}^{2}(\tau)}+\sqrt{E_{2}^{2}(\tau)}\right]
$$

where $\tau$ denotes a complete set of indices and $E_{i}^{2}(\tau)>0, i=1,2$ are the eigenvalues of the modified Lichnerowicz operator

$$
\left(\widehat{\triangle}_{L}^{m} h^{\perp}\right)_{i j}=\left(\triangle_{L} h^{\perp}\right)_{i j}-4 R_{i}^{k} h_{k j}^{\perp}+{ }^{3} R h_{i j}^{\perp}
$$

acting on traceless-transverse tensors of the perturbation and where $\triangle_{L}$ is the Lichnerowicz operator defined by

$$
\left(\triangle_{L} h\right)_{i j}=\triangle h_{i j}-2 R_{i k j l} h^{k l}+R_{i k} h_{j}^{k}+R_{j k} h_{i}^{k},
$$

with $\triangle=-\nabla^{a} \nabla_{a}$. For the background (1), one can define two r-dependent radial wave numbers

$$
k_{i}^{2}\left(r, l, \omega_{i, n l}\right)=\omega_{i, n l}^{2}-\frac{l(l+1)}{r^{2}}-m_{i}^{2}(r) \quad i=1,2,
$$

where 


$$
\left\{\begin{array}{l}
m_{1}^{2}(r)=\frac{6}{r^{2}}\left(1-\frac{b(r)}{r}\right)+\frac{3}{2 r^{2}} b^{\prime}(r)-\frac{3}{2 r^{3}} b(r) \\
m_{2}^{2}(r)=\frac{6}{r^{2}}\left(1-\frac{b(r)}{r}\right)+\frac{1}{2 r^{2}} b^{\prime}(r)+\frac{3}{2 r^{3}} b(r)
\end{array}\right.
$$

are two r-dependent effective masses $m_{1}^{2}(r)$ and $m_{2}^{2}(r)$. When we perform the sum over all modes, $E^{T T}$ is usually divergent. In Refs. [6, 7] a standard regularization/renormalization scheme has been adopted to handle the divergences. In this contribution, we will consider the effect of Gravity's Rainbow on the graviton to one loop. One advantage in using such a scheme is to avoid the renormalization process and to use only one scale: the Planck scale.

\section{Gravity's Rainbow at work and Topology Change}

One of the purposes of Eq. (3) is the possible discovery of a traversable wormhole with the determination of the shape function. When Gravity's Rainbow is taken under consideration, spacetime is endowed with two arbitrary functions $g_{1}\left(E / E_{P}\right)$ and $g_{2}\left(E / E_{P}\right)$ having the following properties

$$
\lim _{E / E_{P} \rightarrow 0} g_{1}\left(E / E_{P}\right)=1 \quad \text { and } \quad \lim _{E / E_{P} \rightarrow 0} g_{2}\left(E / E_{P}\right)=1 .
$$

$g_{1}\left(E / E_{P}\right)$ and $g_{2}\left(E / E_{P}\right)$ appear into the solutions of the modified Einstein's Field Equations [8]

$$
G_{\mu v}\left(E / E_{P}\right)=8 \pi G\left(E / E_{P}\right) T_{\mu v}\left(E / E_{P}\right)+g_{\mu v} \Lambda\left(E / E_{P}\right),
$$

where $G\left(E / E_{P}\right)$ is an energy dependent Newton's constant, defined so that $G(0)$ is the low-energy Newton's constant and $\Lambda\left(E / E_{P}\right)$ is an energy dependent cosmological constant. Usually $E$ is the energy associated to the particles deforming the spacetime geometry. Since the scale of deformation involved is the Planck scale, it is likely that spacetime itself fluctuates in such a way to produce a ZPE. However the deformed Einstein's gravity has only one particle available: the graviton. As shown in Ref.[11], the self sustained equation (3) becomes

$$
\frac{b^{\prime}(r)}{2 G g_{2}\left(E / E_{P}\right) r^{2}}=\frac{2}{3 \pi^{2}}\left(I_{1}+I_{2}\right) \text {. }
$$

Eq.(11) is finite for appropriate choices of the Rainbow's functions $g_{1}\left(E / E_{P}\right)$ and $g_{2}\left(E / E_{P}\right)$. We assume that

$$
g_{1}\left(E / E_{P}\right)=\exp \left(-\alpha E^{2} / E_{P}^{2}\right) \quad g_{2}\left(E / E_{P}\right)=1,
$$

where $\alpha \in \mathbb{R}$ and $g_{2}\left(E / E_{P}\right)=1$, to avoid Planckian distortions in the classical term. We find 


$$
I_{1}=3 \int_{\sqrt{m_{1}^{2}(r)}}^{\infty} \exp \left(-\alpha \frac{E^{2}}{E_{P}^{2}}\right) E^{2} \sqrt{E^{2}-m_{1}^{2}(r)} d E
$$

and

$$
I_{2}=3 \int_{\sqrt{m_{2}^{2}(r)}}^{\infty} \exp \left(-\alpha \frac{E^{2}}{E_{P}^{2}}\right) E^{2} \sqrt{E^{2}-m_{2}^{2}(r)} d E .
$$

Following Ref.[11], after the integration one finds that Eq.(11) can be rearranged in the following way

$$
\frac{b^{\prime}(r)}{2 G r^{2}}=\frac{E_{P}^{4}}{2 \pi^{2}}\left[\frac{x_{1}^{2}}{\alpha} \exp \left(-\frac{\alpha x_{1}^{2}}{2}\right) K_{1}\left(\frac{\alpha x_{1}^{2}}{2}\right)+\frac{x_{2}^{2}}{\alpha} \exp \left(\frac{\alpha x_{2}^{2}}{2}\right) K_{1}\left(\frac{\alpha x_{2}^{2}}{2}\right)\right],
$$

where $x_{1}=\sqrt{m_{1}^{2}(r) / E_{P}^{2}}, x_{2}=\sqrt{m_{2}^{2}(r) / E_{P}^{2}}$ and $K_{1}(x)$ is a modified Bessel function of order 1 . Note that it is extremely difficult to extract any useful information from this relationship, so that in the following we consider two regimes, namely the cis-planckian regime, where $x_{i} \ll 1(i=1,2)$, and the trans-planckian régime, where $x_{i} \gg 1$. In Ref.[11], it has been shown that the cis-planckian regime does not produce solutions compatible with traversability. On the other hand when we fix our attention on the trans-planckian regime, i.e., $x_{1} \gg 1$ and $x_{2} \gg 1$, we obtain the following approximation

$$
\frac{1}{2 G} \frac{b^{\prime}(r)}{r^{2}} \simeq \frac{E_{P}^{4}}{8 \sqrt{\alpha^{3} \pi^{3}}}\left[\exp \left(-\alpha x_{1}^{2}\right) x_{1}+O\left(\frac{1}{x_{1}}\right)+\exp \left(-\alpha x_{2}^{2}\right) x_{2}+O\left(\frac{1}{x_{2}}\right)\right] .
$$

Note that in this regime, the asymptotic expansion is dominated by the Gaussian exponential so that the quantum correction vanishes. Thus, the only solution is $b^{\prime}(r)=0$ and consequently we have a constant shape function, namely, $b(r)=r_{t}$. It is interesting to observe that Eq.(11) can be interpreted also in a different way. Indeed, if we fix the background on the r.h.s. of Eq.(11) and consequently let the quantum fluctuations evolve, one can verify what kind of solutions it is possible to extract from the 1.h.s. in a recursive way. In this way, if we discover that the 1.h.s. has solutions which topologically differ from the fixed background of the r.h.s., we can conclude that a topology change has been induced from quantum fluctuations of the graviton for any spherically symmetric background on the r.h.s of Eq. (11). Of course, this is not a trivial task, therefore the simplest way to see if a topology change is realized, we fix the Minkowski background on the r.h.s. of Eq. (11). This means that $b(r)=0 \forall r$ and the effective masses become $m_{1}^{2}(r)=m_{2}^{2}(r)=6 / r^{2}$. Then Eq. (15) reduces to

$$
\frac{1}{2 G} \frac{b^{\prime}(r)}{r^{2}}=\frac{E_{P}^{4}}{\pi^{2}}\left[\frac{6}{\alpha\left(r E_{P}\right)^{2}} \exp \left(-\frac{3 \alpha}{\left(r E_{P}\right)^{2}}\right) K_{1}\left(\frac{3 \alpha}{\left(r E_{P}\right)^{2}}\right)\right] .
$$

Let us fix our attention on the trans-planckian regime, i.e., $r E_{P} \ll 1$, where we can write 


$$
\frac{b^{\prime}(r)}{r^{2}} \simeq \frac{E_{P}^{2}}{2 \sqrt{\alpha^{3} \pi^{3}}}\left[\exp \left(-\alpha \frac{6}{\left(r E_{P}\right)^{2}}\right) \frac{\sqrt{6}}{r E_{P}}+O\left(r E_{P}\right)\right] .
$$

Since this is a particular case of Eq.(16), we conclude that the only solution is $b^{\prime}(r)=0$ and consequently we have a constant shape function, namely, $b(r)=r_{t}$. A comment to the result (18) is in order. One could think that Eq.(18) is only a special case of Eq. (16). Of course this is not true, because Eq. (16) uses a different initial condition with respect to Eq.(18). Indeed, in Eq. (16) the background is arbitrary, while in Eq. (18) one considers a Minkowski line element and the solution is obtained with an iterative process. One can observe that this procedure could be approached also distorting the one loop graviton by means of a Noncommutative geometry like in Ref.[12, 13], where the classical Liouville measure is modified into[12]

$$
d n_{i}=\frac{d^{3} \mathbf{x} d^{3} \mathbf{k}}{(2 \pi)^{3}} \exp \left(-\frac{\theta}{4}\left(\omega_{i, n l}^{2}-m_{i}^{2}(r)\right)\right), \quad i=1,2 .
$$

$m_{i}^{2}(r)$ are the effective masses described in $(8)$ and $\theta$ is the Noncommutative parameter. While nothing can be said about the effect of Noncommutative geometry on topology change, a result can be extracted from the traversability of the wormhole. Indeed, if one fixes the form of the shape function to be $b(r)=r_{0}^{2} / r$, which is the prototype of the traversable wormholes[3], one gets $r_{0}=0.28 l_{P}$, with $\theta$ fixed at $\theta=7.43 \times 10^{-2} l_{P}^{2}$. If we compare the result obtained in Ref.[11] using Gravity's Rainbow, one finds the following value for the radius $r_{t}=1.46 l_{P}$, which is slightly larger than $r_{0}$. The conclusion is that Gravity's Rainbow and Noncommutative geometry keep under control the UV divergences in this ZPE calculation connected with the self-sustained equation. In both cases we find that the result is a Wheeler wormhole. This means that, from the point of view of traversability, the wormhole will be traversable in principle, but not in practice.

\section{References}

1. A. Einstein and N. Rosen, Phys. Rev. 48, 73, (1935).

2. J. A. Wheeler, Phys. Rev. 97, 511-536 (1955).

3. M. S. Morris and K. S. Thorne, Am. J. Phys. 56, 395 (1988)

4. A. DeBenedictis, R. Garattini and F. S. N. Lobo, Phys. Rev. D 78, 104003 (2008); arXiv:0808.0839 [gr-qc].

5. Visser M 1995 Lorentzian Wormholes: From Einstein to Hawking (American Institute of Physics, New York).

6. R. Garattini, Class. Quant. Grav. 22, 1105 (2005); arXiv:gr-qc/0501105

7. R. Garattini, Class. Quant. Grav. 24, 1189 (2007); arXiv: gr-qc/0701019 R. Garattini and F. S. N. Lobo, Class. Quant. Grav. 24, 2401 (2007); arXiv:gr-qc/0701020

8. J. Magueijo and L. Smolin, Class. Quant. Grav. 21, 1725 (2004); arXiv:gr-qc/0305055

9. R. Garattini and F. S. N. Lobo, Phys. Lett. B 671, 146 (2009); $\operatorname{arXiv:0811.0919~[gr-qc].~}$

10. R. Garattini and G. Mandanici, Phys. Rev. D 83, 084021 (2011). arXiv:1102.3803 [gr-qc].

11. R. Garattini and F. S. N. Lobo, Phys. Rev. D 85 (2012) 024043; ArXiv: 1111.5729 [gr-qc].

12. R. Garattini and P. Nicolini, Phys. Rev. D 83, 064021 (2011); arXiv:1006.5418 [gr-qc].

13. R. Garattini, EPJ Web Conf. 5801007 (2013); arXiv:1212.4311 [gr-qc] 